\title{
Wireless Control System for Six-Legged Autonomous Insect Robot
}

\author{
Sim Kok Swee, Amro Al-Qudah \\ MMU University, Faculty of Engineering \& Technology, 75450 Melaka, Malaysia
}

\begin{abstract}
Insect robots are a special type of robots that designed to imitate the behavior of insects. Insect robots have many advantages such as the ability to move over uneven terrain, less power consumption and smaller in size. This paper shows the progress made during the development of a six-legged robot system inspired by ants and crickets. The resulted robot is able to mimic insects in terms of gait pattern and physical size. The robot is controlled wirelessly by using a Bluetooth xBee module and remote devices including a mobile phone with android application, a personal computer with windows software, and a Bluetooth wireless controller made the Arduino development platform.
\end{abstract}

\section{Introduction}

Improvements made in robotics technology have spurred our economy and changed our lives [1]. Robots are perfect for tasks that need repetitive precise movements or considered dangerous for humans [2]. In manufacturing, robots have improved the quality of the product, increased production output rates and reduced operating costs [3]. Mobile robot is a device that can move autonomously from place to place to achieve a set of goals [4]. Mobile robots are used in a wide range of applications including military operations, manufacturing, search and rescue, security, health care (e.g. pharmaceutical delivery), and home applications (e.g. robotic vacuum cleaner) [4]. Insect robots are that special type of mobile robots that imitate the insects in terms of gait pattern and physical size [5]. Designs of legged and mobile robots have been influenced by insects because of their great mobility and ability to move over uneven terrain. [6]. Insect robot based manufacturing systems have less power consumption and less vibration due to its small size [7]. Insect robots can access places where human cannot such as hazardous places or small spaces. It has an advantage over wheeled robots in moving over uneven terrain [8] [9]. Wirelessly controlled robots eliminate the need of wiring and they have a longer range, which eliminate the need of people in hazardous places [10].

For insect robots, mechanical energy consumption is mainly divided into three portions; energy to support robot weight, energy to produce traction and energy to oscillate the legs [11]. In order to achieve an optimum usage of energy, the insect robot should travel with reduced duty factor, the height of the body should be maximum, step length should be as short as possible, and deviation of each leg to slip should be as low as possible [11].

The hexapod robot has inspired by insects such as ants and crickets. Having six legs, it gives the ability to move flexibly uneven various terrains and does not require any balancing mechanisms to stand upright [12].

\section{Problem formulation}

Autonomous walking robot is a potential research subject [13]. At the present, insect robots still face many limitations such as slow moving speed, complex mechanism and complicated control system [14]. Insect robots are a great tool to access places which are dangerous for human such as nuclear reactors, outside the atmosphere and even behind enemy lines in the war. Wireless commutations add several advantages to insect robots for instance, eliminate wiring and make it easier to replace people in risky areas [15].

\section{Hardware and Software Design}

In this experiment, the insect inspired robot is controlled using a PIC microcontroller unit which drives three micro-servomotors. The microcontroller receives orders from a Bluetooth module. On the other hand the Bluetooth module communicates with software installed on a Bluetooth-enabled mobile phone. Furthermore, operator commands can also be sent from PC computer or Bluetooth remote controller. An adjustable infrared sensor is used to discover obstacles. The robot is battery powered to eliminate the need of wiring. 


\subsection{Hardware Design}

To make the insect robot lightweight and considerably strong, $3 \mathrm{~mm}$ acrylic sheet is used to form the main body of the robot. Body dimensions are $(75 \times 45 \times 3) \mathrm{mm}$. The legs are constructed using a stainless steel with $1 \mathrm{~mm}$ thickness. To increase stability, the end of the legs is made into a shape of compression coil spring; this construction is able to absorb shocks and vibrations. Nylon cable is used to make fasteners. The front legs and back legs of each side are connected by a stainless steel link.

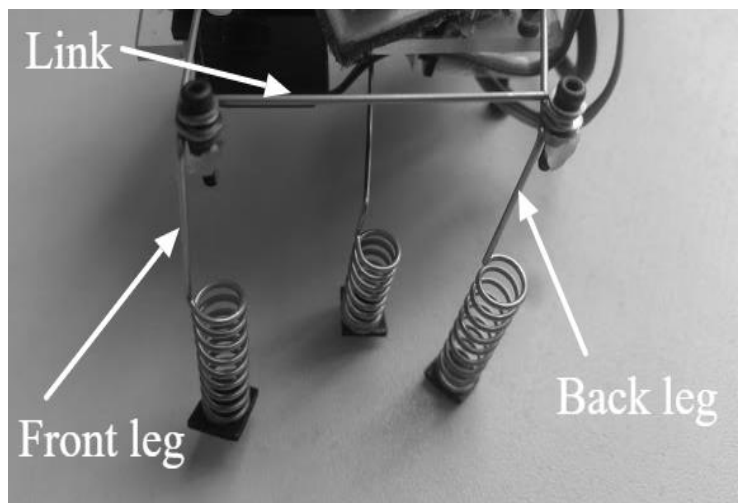

Figure 1.Stainless steel links connect the front and back legs.

PIC18F252 microcontroller has been selected for the insect robot. PIC18F252 is a powerful small 28 pin microcontroller that has enough $\mathrm{I} / \mathrm{O}$ pins to take over the servomotors, the IR switch and the Bluetooth module. The microcontroller is clocked at $20 \mathrm{MHz}$ using a crystal oscillator with two $330 \mathrm{pF}$ ceramic capacitors.

An IR switch is used to detect and avoid obstacles that are in the front of the insect robot. The range of the IR switch is adjustable from $3 \mathrm{~cm}$ to $80 \mathrm{~cm}$ using a switch in the rear of the sensor. The range is also affected by the color and surface of the obstacles. Usually white color obstacles are detected from longer range than dark obstacles. The sensor operates at $5 \mathrm{VDC}$ and consumes up to $100 \mathrm{~mA}$. The IR sensor internally cancels interferences caused by sunlight or bulbs. The output of the IR sensor will be low when an obstacle is detected.

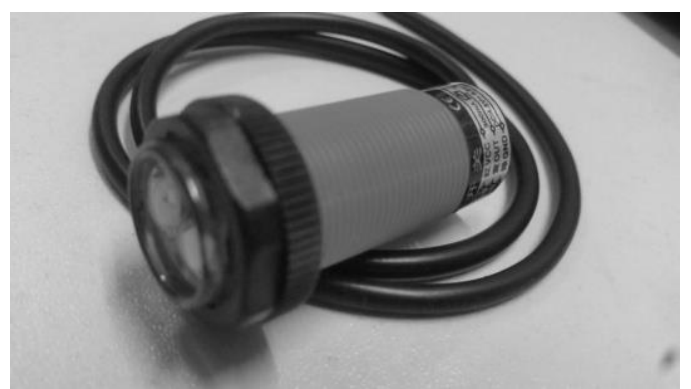

Figure 2. An adjustable infrared sensor switch.

Three servomotors (Power HD-1550A) are used to drive the robot. The dimension of this servomotor is $(22 \times 11 \times 20.1) \mathrm{mm}$ which makes it suitable for a small insect robot. The stall torque of the servomotor is $0.8 \mathrm{~kg}$ $\mathrm{cm}$ at $4.8 \mathrm{~V}$, at the same voltage with no load the speed is $0.12 \mathrm{Sec} / 60$ degree.

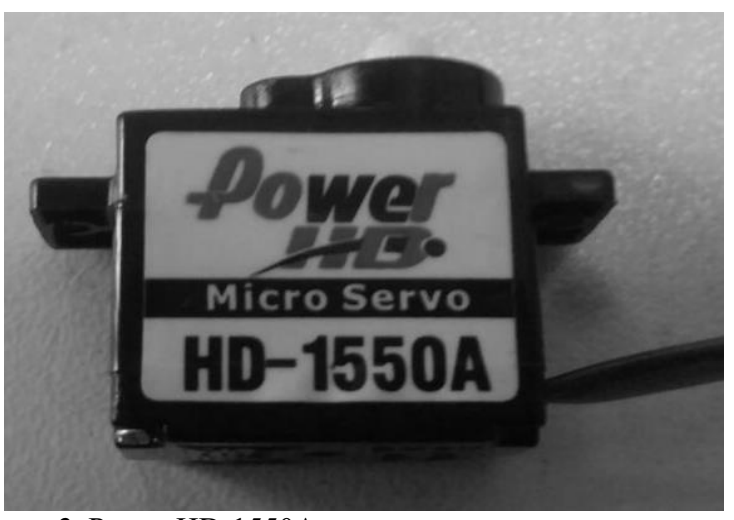

Figure 3. Power HD-1550A servomotor.

A rechargeable dual cell Lithium Polymer (LiPo) battery is chosen to power up the robot. The output voltage of the battery is $7.4 \mathrm{VDC}$ and the capacity is about $900 \mathrm{mAH}$. Voltage regulator L7805 is used to provide constant $5 \mathrm{~V}$ for the microcontroller. The dimensions of the battery are $(55 \times 29 \times 14) \mathrm{mm}$.

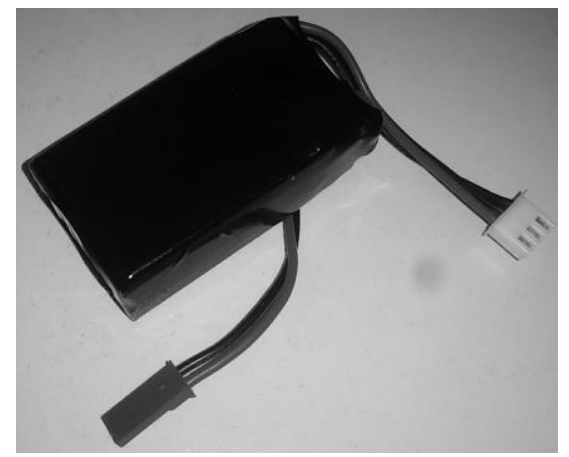

Figure 4. LIPO 7.4 VDC - 900mAH Battery.

Bluetooth module XBee is chosen as robot receiver. Also, another XBee module is used in the remote controller as the transmitter. XBee is compact in size and have a $5 \mathrm{~V}$ power out. The XBee module has a USB mini b-type interface used to download the XBee firmware and configurations. The Bluetooth module board has 5 pins which are: $5 \mathrm{~V}$ power source voltage, ground pin, $\mathrm{R}_{\mathrm{X}}, \mathrm{T}_{\mathrm{X}}$ and reset pin. Reset pin is optional as there is already a reset button on XBee board itself. Pins $5 \mathrm{~V}$ and ground are connected directly to the ground pin and $5 \mathrm{~V}$ pin of the microcontroller while $\mathrm{R}_{\mathrm{X}}$ and $\mathrm{T}_{\mathrm{X}}$ of $\mathrm{XBee}$ and $\mathrm{R}_{\mathrm{X}}$ and $\mathrm{T}_{\mathrm{X}}$ of PIC18F252 are cross connected. Bluetooth module in the insect robot acts as a slave, while in the remote controller acts as the master. XBee module is connected to the mainboard through SKXBee. This board is basically a USB to UART board for the XBee module board. 


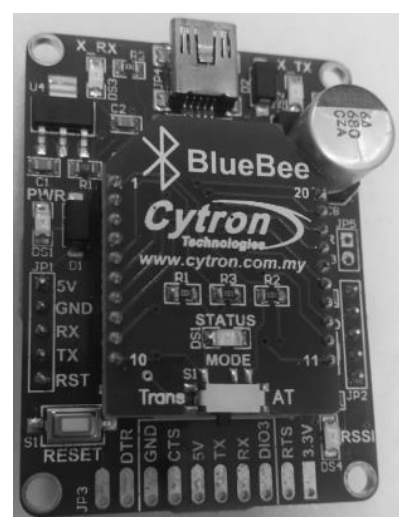

Figure 5. XBee Bluetooth module.

The Bluetooth remote controller has been constructed using Arduino UNO board plus an input shield with an XBee module mounted on the top of it. The controller is powered by a $9 \mathrm{~V}$ battery.

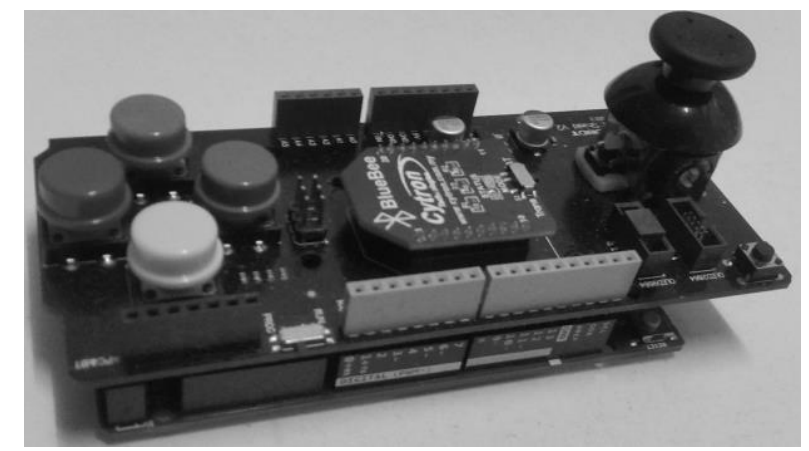

Figure 6. Bluetooth remote controller.

The main board is designed to be compacted in size; the dimensions are $55 \mathrm{~mm}(\mathrm{~L}) \times 61 \mathrm{~mm}(\mathrm{~W})$.

\subsection{Software Design}

For schematic diagram design the application ExpressSCH is used. ExpressSCH is a free CAD software that is easy to use [16]. However, ExcpressSCH does not have PCB layout design features, so the PCB has been designed using software called Traxmaker [17].

MikroC PRO for PIC is a full-featured integrated user environment [18] used to develop firmware for the insect robot. The firmware is coded in ANSI C programming language.

After compiling and building the firmware for the robot, PicKit 2 programmer software has been used to download the binaries to the PIC microcontroller. PicKit software is free, but requires an ICSP interface [18].

The Arduino Software [19] is the integrated development environment that was used to program the Bluetooth controller. Arduino IDE provides code editor, debugger, compiler, downloader and also a real-time monitor which is useful to check the data sent by the controller.

XBee modules are not easy to configure. The software $\mathrm{XCTU}$ is a Windows application developed to enable interactions with XBee modules through a simple graphical interface. XCTU includes a set of tools that made it easy to set-up, configure and test XBee models. [20]

Microsoft Visual C++ [21] is a free IDE that has enabled us to develop a windows software to control the robot. Control signals are sent from the application to a logical COM port that represents the Bluetooth adapter inside the PC.

\subsection{Methodology}

The main advantage of hexapod robots is the ability to move faster with a relatively high stability. That stability can be maintained by keeping three legs on the ground at the same time [6]. Table 1 shows a sample sequence of leg movements that form the gait in our robot. White color indicates that the leg is in the air while the Gray color indicates the leg in touch with the ground.

Table 1. Robot gait pattern

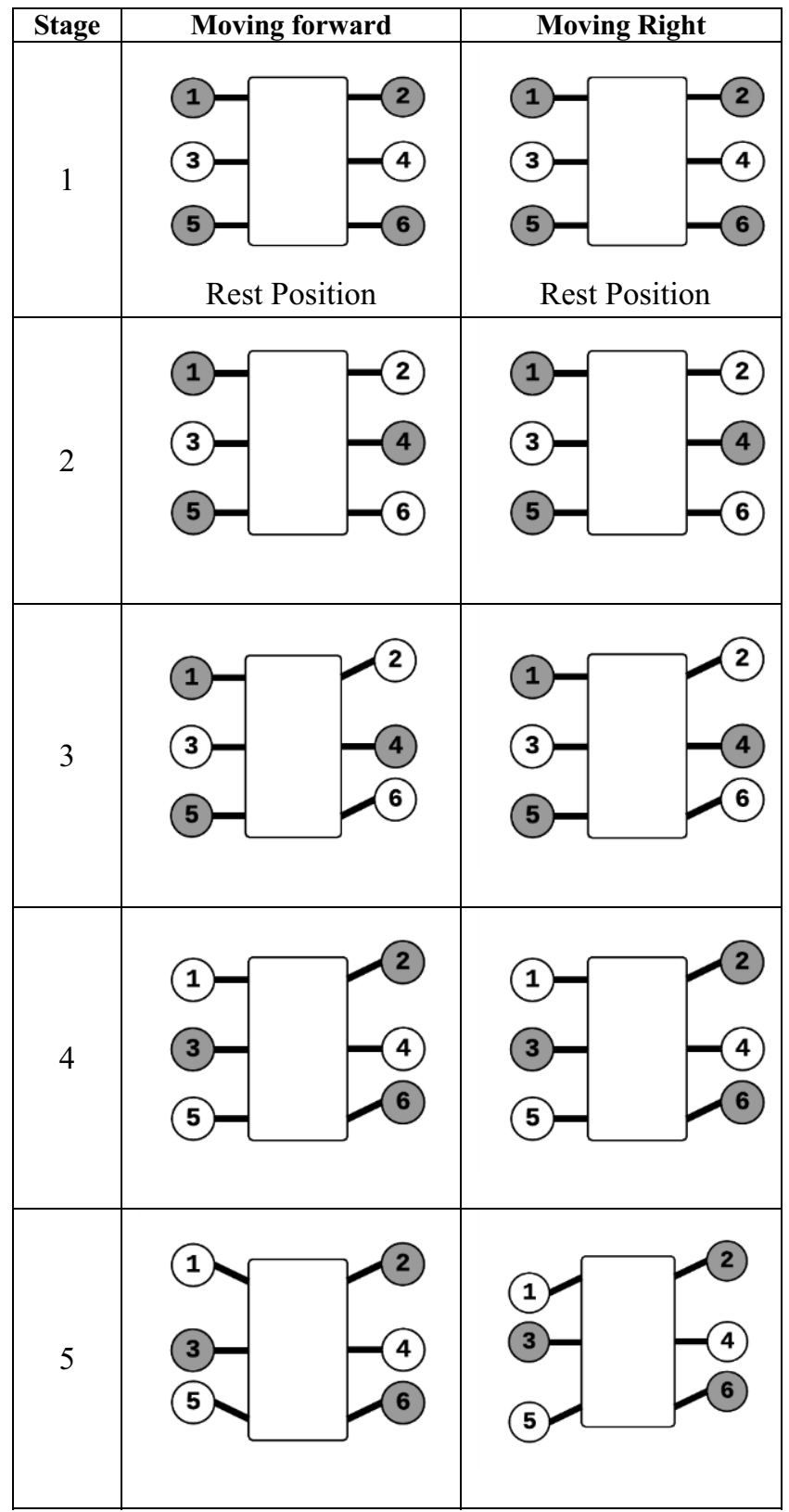




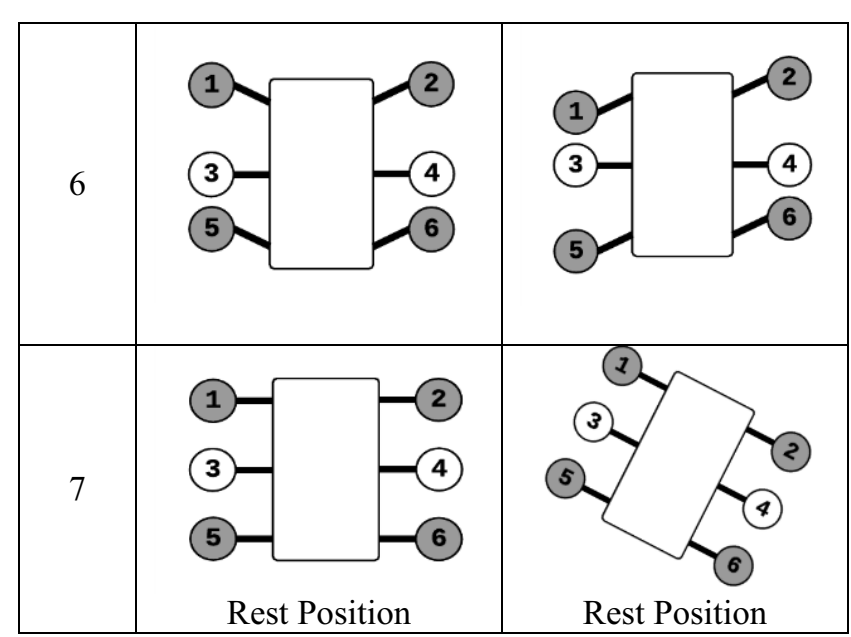

The microcontroller firmware receives inputs from IR sensor as well as the XBee Bluetooth module. The outputs are three control signals for the servomotors. The next figure shows the main flow diagram of the program.

The XBee module in the Bluetooth remote controller is converted to master mode in order to able to start communications and control the robot. The remote controller has pushbuttons each represents a direction of movement in the robot.

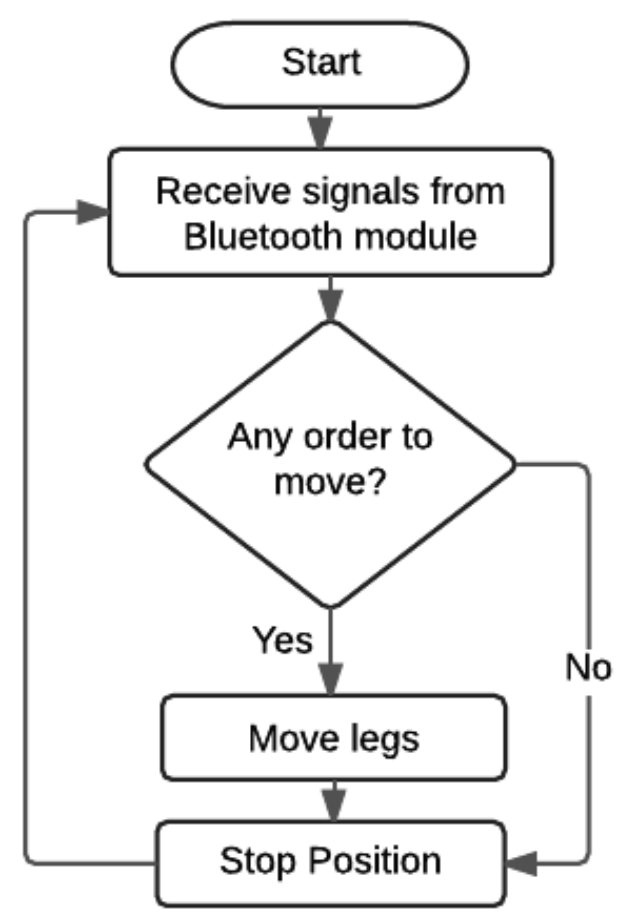

Figure 7. Main program flow diagram.

\section{Results}

The developed insect robot shown in figure 8 is able to mimic the behavior of insects. Infrared sensor helps the robot to avoid obstacles. The robot is lightweight and the final size of the robot is about $(13 \times 9 \times 7) \mathrm{cm}$.

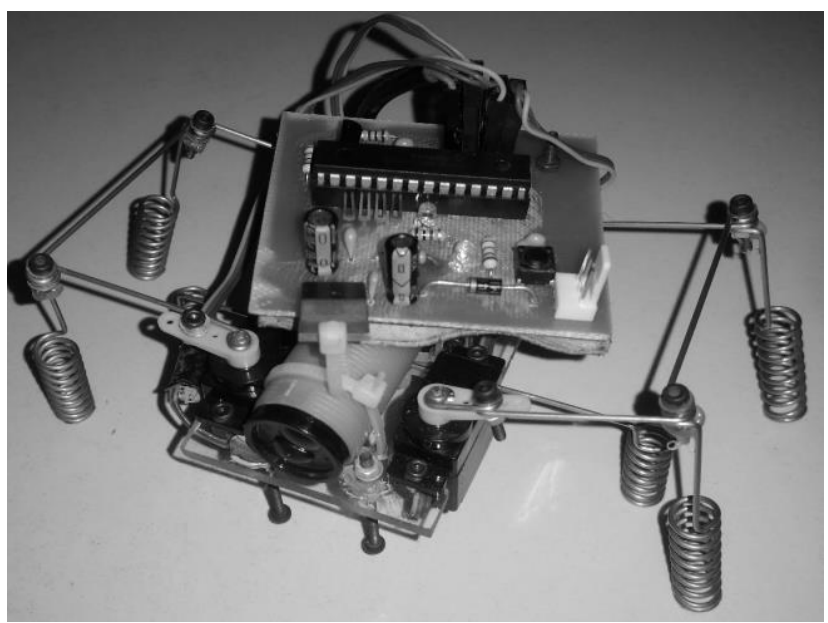

Figure 8. Six-legged autonomous insect robot.

\section{References}

1. J. F. Engelberger, Robotics in Practice: Management and Applications of Industrial Robots. (1980)

2. A. Tayebi, "Adaptive iterative learning control for robot manipulators," Automatica, 40, 1195-1203, (2004)

3. Ifr, "History of Industrial Robots From the first installation until today," International Federation of Robotics, (2012)

4. S. Tzafestas, Introduction to Mobile Robot Control. Elsevier, (2013)

5. A. Piore, "Rise Of The Insect Drones," Popular Science, (2014)

6. W. a. Lewinger and R. D. Quinn, "A hexapod walks over irregular terrain using a controller adapted from an insect's nervous system," IEEE/RSJ 2010 Int. Conf. Intell. Robot. Syst. IROS 2010 - Conf. Proc., 3386-3391, (2010)

7. O. Fuchiwaki, "Insect-sized holonomic robots for precise, omnidirectional, and flexible microscopic processing: Identification, design, development, and basic experiments," Precis. Eng., 37, 88-106, (2013)

8. B. G. a Lambrecht, A. D. Horchler, and R. D. Quinn, "A small, insect-inspired robot that runs and jumps," Proc. - IEEE Int. Conf. Robot. Autom., 1240-1245, (2005)

9. R. Quinn, G. Nelson, R. Bachmann, D. Kingsley, J. Offi, and R. Ritzmann, "Insect designs for improved robot mobility," Int Conf Climbing Walk. Robot., 69-76, (2001)

10. I. Firmansyah, B. Hermanto, and L. T. Handoko, "Control and Monitoring System for Modular Wireless Robot.”, (2007)

11. M. F. Silva and J. T. Machado, "A literature review on the optimization of legged robots," J. Vib. Control, 18, 1753-1767, (2012)

12. K. Aparna and S. Geeta, "Insect Inspired Hexapod Robot for Terrain Navigation," 63-69, (2013) 
13. B. Klaassen, R. Linnemann, D. Spenneberg, and F. Kirchner, "Biomimetic walking robot SCORPION: Control and modeling," Rob. Auton. Syst., 41, 69$76,(2002)$

14. R. D. Beer, R. D. Quinn, H. J. Chiel, and R. E. Ritzmann, "Biologically inspired approaches to robotics: what can we learn from insects?," Commun. ACM, 40, 30-38, (1997)

15. R. Calcagno, F. Rusin, F. Deregibus, A. S. Vincentelli, and A. Bonivento, "Application of wireless technologies in automotive production systems," Vdi Berichte, 57, (2006)

16. ExpressPCB LLC, "ExpressSCH Software." [Online].Available: http://www.expresspcb.com. [Accessed: 27-Aug-2015].

17. MicroCode, "Traxmaker Software." [Online]. Available: http://microcode.com. [Accessed: 27Aug-2015].

18. mikroElektronika, "mikroC for PIC." [Online]. Available: http://www.mikroe.com. [Accessed: 27Aug-2015].

19. Arduino LLC, "Arduino Software IDE." [Online]. Available: http://www.arduino.cc. [Accessed: 27Aug-2015].

20. Digi International, "XCTU software." [Online]. Available: http://www.digi.com. [Accessed: 27Aug-2015].

21. "Microsoft Visual C ++," Microsoft, 1989. [Online]. Available: http://msdn.microsoft.com [Accessed: 27-Aug-2015]. 